\title{
Calidad en la atención en el Sistema de Salud en Bucaramanga y su área metropolitana
}

\author{
Assistance quality in the health system in \\ Bucaramanga and its metropolitan area
}

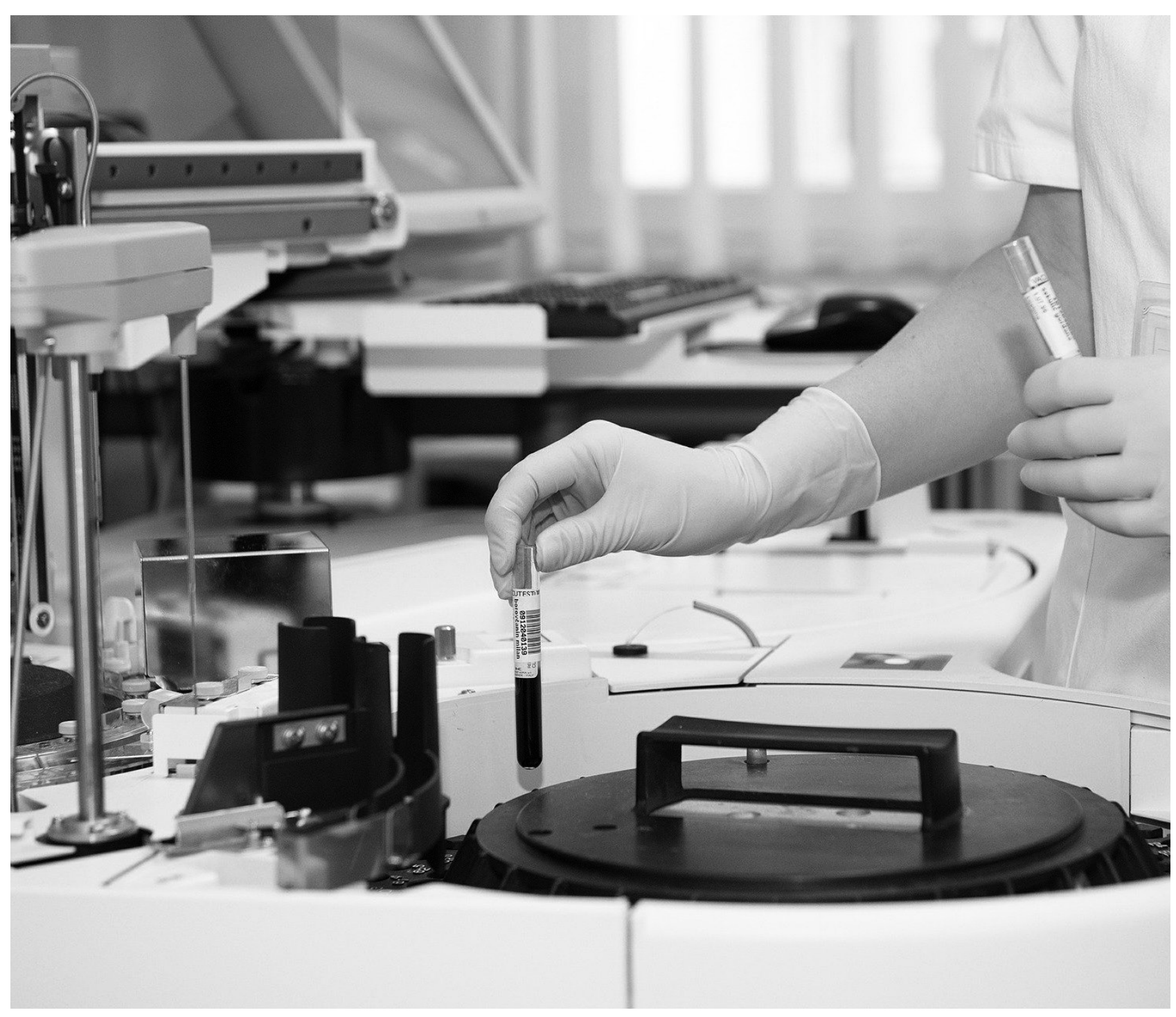




\section{Calidad en la atención en el Sistema de Salud en Bucaramanga y su área metropolitana ${ }^{1}$ Assistance quality in the health system in Bucaramanga and its metropolitan area}

\section{Diana Oliveros Contreras², Mauricio Mendoza García³ , Alba Patricia Guzmán Duque ${ }^{4}$}

Artículo recibido en marzo 14 de 2019; artículo aceptado en mayo 15 de 2019.

Este artículo puede compartirse bajo la Licencia Creative Commons Atribución-NoComercial-Compartirlgual 4.0 Internacional y se referencia usando el siguiente formato: Oliveros, D., Mendoza, M. \& Guzman, A. (2019). Calidad en la atención en el Sistema de Salud en Bucaramanga y su área Metropolitana. I+D Revista de Investigaciones, 14 (2), 113-121.

DOI: https://doi.org/10.33304/revinv.v14n2-2019010

\section{Resumen}

El objetivo de este estudio fue analizar la calidad en los servicios de salud en Bucaramanga y su área metropolitana, durante el periodo 2011-2015, a través de 17 indicadores del Ministerio de Salud de Colombia. En estos indicadores, la dimensión de la calidad presenta tres cualidades definidas: 1) efectividad, 2) seguridad y 3) experiencia de la atención. Se encontraron problemáticas en la calidad del uso de los recursos destinados a la salud en Bucaramanga y su área metropolitana, que están relacionados con tales indicadores. Bucaramanga y Floridablanca, por término medio, son los municipios con mayores dificultades relacionados con tasas de oportunidad en la asignación de citas médicas en las distintas especialidades, así como las tasas de mortalidad de los indicadores evaluados. Esto se debió principalmente a las condiciones geográficas y la ubicación del mayor número de clínicas y centros hospitalarios.

Palabras clave: Calidad, salud, gestión, seguridad, satisfacción del usuario.

\footnotetext{
Abstract

The aim of this study was to analyze the quality of health services in Bucaramanga and the metropolitan area during the period 2011-2015, through 17 indicators of the Ministry of Health of Colombia, where the quality dimension presents three defined qualities: 1) effectiveness, 2) safety and 3) experience of care. Problems were found in the

1. Artículo de investigación, de tipo no experimental, cuantitativo de corte longitudinal con un diseño descriptivo, resultado de un proyecto de investigación culminado, perteneciente al área de organizaciones, subárea de estrategia, desarrollado en el grupo Estratégico de Investigación Organizacional GENIO, financiado por la Universidad Autónoma de Bucaramanga (Colombia). Dirección: avenida 42 n. ${ }^{\circ} 48-11$, PBX: 57) (7) 643 6111/643 6261. Fecha de inicio: 30 de septiembre de 2015. Fecha de terminación: 30 de junio de 2017.

2. Vinculado al Grupo Estratégico de Investigación Organizacional GENIO, Universidad Autónoma de Bucaramanga (Colombia): Dirección: avenida 42 n. 48-11, PBX: (57) (7) 6436111/6436261. ORCID ID: https://orcid.org/0000-0001-9641-7772. Correo electrónico institucional: doliveros364@ unab.edu.co.

3. Vinculado al Grupo Estratégico de Investigación Organizacional GENIO, Universidad Autónoma de Bucaramanga (Colombia): Dirección: avenida 42 n.o 48-11, PBX: (57) (7) 6436111/6436261. ORCID ID: http://orcid.org/0000-0002-0652-3027. Correo electrónico institucional: m.mendoza@unab. edu.co.

4. Vinculado al Grupo Estratégico de Investigación Organizacional GENIO, Universidad Autónoma de Bucaramanga (Colombia): Dirección: avenida 42 n. ${ }^{\circ} 48-11$, PBX: (57) (7) 6436111/6436261. ORCID ID: https://orcid.org/0000-0003-1704-6884. Correo electrónico institucional: aguzman201@ unab.edu.co.
} 
quality of use of resources destined for health in Bucaramanga and the metropolitan area, which are related to such indicators. Bucaramanga and Floridablanca, on average, are the municipalities with greater difficulties related to opportunity rates in the assignation of medical appointments in the different specialties, as well as the mortality rates of the evaluated indicators. This was mainly due to geographical conditions and location of the largest number of clinics and hospitals.

Keywords: Quality, health system, management, security, user satisfaction.

\section{Introducción}

La salud es un aspecto determinante en el bienestar de las personas y una necesidad indispensable para alcanzar un desarrollo humano sostenible. Es evidente que todos los ciudadanos tienen derecho a un cuidado equitativo, eficiente y atento de su salud. El Estado debe garantizar la cobertura en servicios de alta calidad (UNESCO, 2005). La Agenda 2030 para el Desarrollo Sostenible (ODS) establece una visión transformadora hacia la sostenibilidad económica, social y ambiental. Precisamente, existe una hoja de ruta para los 193 países que se suscribieron, en la que se incluyen temas prioritarios para la región: acabar con la pobreza extrema; garantizar una vida sana y la promoción del bienestar de todos; reducir la desigualdad en todas sus dimensiones, entre otros (CEPAL, 2018).

Si bien diferentes gobiernos declaran como principio la cobertura universal de la salud, la seguridad social y la calidad de los servicios, existen dificultades para alcanzarlos. En algunos países se presenta el problema de cómo afiliar a todas las personas a la seguridad social, considerando los limitados recursos que existen y que se requieren para financiar y garantizar los beneficios de cobertura universal (Zea \& Restrepo, 2002).

A partir de 1990, las economías latinoamericanas en el campo de la salud atravesaron por reformas estructurales y sectoriales. La seguridad social sufrió transformaciones importantes que buscaban la mejora de la eficiencia de los sistemas de salud, la calidad en los servicios y la sostenibilidad a largo plazo. Precisamente, a pesar de la pluralidad de cada uno de los países en relación con el desarrollo, y en especial, la situación del sector salud, existen denominadores comunes entre países: (1) la necesidad de disminuir las inequidades en salud, derivadas de factores como la pobreza, género, la raza y/o edad; (2) avances en la protección social en salud, para ampliar la cobertura; (3) la obligación de incrementar la inversión de gasto público corriente y del sector; (4) la reorientación de los servicios de atención de la salud basados en la participación activa de todos los usuarios del sistema; (5) la necesidad de un fortalecimiento de la infraestructura en salud pública; y (6) la necesidad de avanzar en el diseño y aplicación de políticas intersectoriales que incidan sobre las metas de salud (UNESCO, 2005).

En los últimos años, Colombia ha realizado un esfuerzo para que todos los ciudadanos accedan en condiciones de equidad y calidad de forma rápida y oportuna a los servicios de salud. Sin embargo, pese a los progresos del sistema, se evidencia inconformidad de los ciudadanos, por la atención recibida en algunas entidades, las limitaciones de acceso, la falta de oportunidad en la recepción de los servicios, entre otros aspectos (MINSALUD, 2009). En el país, la reforma a la seguridad social fue discutida, promulgada y consagrada en la Constitución de 1991, en la que el Estado presenta la salud como un derecho fundamental de las personas, de forma que deja de ser un favor brindado por el Estado para convertirse en una obligación. Posteriormente, la Ley 100 de 1993 consagra el Sistema de Seguridad Social en Salud, que pasa a tener como principios fundamentales la universalidad, la solidaridad y la eficiencia. Así, se establece como factor crítico la calidad en la atención y los mecanismos de medición para los proveedores en salud (Roldán, Vargas \& Giraldo, 2001).

El propósito de este estudio fue analizar, durante el periodo 2011-2015, los indicadores de monitoreo del Sistema Obligatorio de Garantía de Calidad de la Atención en Salud en Colombia, de acuerdo con la Resolución N. ${ }^{\circ} 1446$ del 8 mayo de 2006, expedida por el Ministerio de la Protección Social. Esta resolución propone acciones de mejora que requieren incluirse en la política pública, para mejorar la calidad en el servicio de salud de los colombianos.

\section{Calidad del Sistema de Salud}

La calidad de vida de la población está relacionada con el adecuado funcionamiento de los sistemas de salud. Un sistema de salud es bueno si responde a las perspectivas de los usuarios (Kruk et al., 2018); y es equitativo si incluye a todos sin discriminación (Charnes, 2013). Para Calderón (2019), los principales retos de la atención primaria de salud para ser integradora y de calidad son integralidad en atención al usuario, coordinación y colaboración entre los actores y monitoreo y seguimiento. 
Existen diversos estudios relacionados con la calidad en el sistema de salud. Murray y Frenk (2000) afirman que los resultados del sistema de salud están basados en tres metas que llevan al mejoramiento de la salud de la población y que deben considerarse como parte de la política pública: capacidad de respuesta del sistema de salud, la justicia en el financiamiento y el riesgo. El objetivo es garantizar que los hogares con menos ingresos no deban sufragar un mayor porcentaje de su gasto discrecional en salud que los hogares con más ingresos; asimismo, que todos los ciudadanos se encuentren resguardados frente a las pérdidas financieras catastróficas relacionadas con enfermedades. Chisholm y Evans (2010) plantean que la eficiencia en el sector salud debe estar dirigida hacia la maximización de los recursos disponibles considerando la protección financiera y la capacidad de respuesta en la prestación del servicio.

Ramírez-Sánchez y Nájera-Aguilar (1998) analizaron la calidad de la atención y servicio recibido por los pacientes en servicios ambulatorios de salud en México. El $81,2 \%$ de los pacientes afirmó que la atención recibida fue satisfactoria, debido al personal y restablecimiento de su salud; mientras que para el 18,8 \% la percepción de mala calidad se generó por el tiempo de espera y la insuficiencia en las labores de examen y diagnóstico. Lo anterior evidencia que la eficiencia no es el único objetivo de un sistema de salud. Es posible que los países sacrifiquen el nivel de eficiencia buscando alcanzar otras metas, como la protección financiera frente al riesgo, o mejoras en la equidad en el ingreso a los servicios de salud y mejora de la calidad en el servicio prestado (Kruk et al., 2018).

Según Cotlear et al. (2015), hay diferentes percepciones frente a la salud en Latinoamérica. Por una parte, se destaca el gran avance en las condiciones de salud y el fortalecimiento de la inversión en la salud al desarrollo económico. Por otra parte, persiste el pensamiento de injusticia relacionado con la segregación social de los sistemas de salud.

Por otro lado, algunos estudios evalúan el sistema de salud en Colombia con relación a eficiencia y calidad, lo que evidencia limitaciones desde el ingreso y la búsqueda de la atención en salud; conflictos en las autorizaciones; largos tiempos de espera para consultas especializadas; procedimientos quirúrgicos y entrega de medicamentos (Hernández, Rubiano \& Barona, 2015); dificultades en referentes a cobertura; calidad y eficiencia (Diaz, Ospina \& Lopez, 2015). Asimismo, Ruiz, Duque y Redondo (2017) concluyen que existe inequidad en el servicio de salud, lo que está relacionado directamente con las condiciones socioeconómicas de la población, la ubicación, y en especial la desigualdad en los ingresos. Echeverri (2008) comentó que administrar los servicios de salud como un producto en una economía de mercado no contribuye al fortalecimiento de la salud, porque conduce a la exclusión y a la inequidad para los usuarios. Mientras que Cano, Pineda y Pulido (2016), a partir del estudio aplicado a usuarios del régimen subsidiado habitantes de la comuna 16 de Medellín, Colombia, concluyeron que para los usuarios son primordiales el trato humanizado, la equidad y la participación.

\section{Metodología}

\section{Tipo de estudio}

El estudio es de tipo no experimental, cuantitativo, de corte longitudinal, con un diseño descriptivo, basado en el análisis de los resultados y el comportamiento de la serie en el tiempo, para los 17 indicadores del Ministerio de Salud de Colombia (MINSALUD, 2009, 2015).

La dimensión de la calidad presenta tres cualidades definidas: 1) efectividad, 2) seguridad y 3 ) experiencia de la atención:

1) Efectividad: Evalúa si el país presenta calidad requerida en atención en salud que contribuya a un incremento en las necesidades de salud de las personas (ver Tabla 1).

2) Seguridad: La atención en los servicios de salud requiere la mayor seguridad posible; por tanto, los siguientes indicadores permiten medir y fomentar la seguridad en salud en los contextos institucionales y profesionales en los que se proporcionan los servicios (ver Tabla 2).

3) Experiencia de la atención: Es un pilar en la prestación de los servicios; las personas estiman la calidad de la experiencia que reciben (ver Tabla 3).

\section{Participantes}

La población la constituyó las IPS de Bucaramanga y su área metropolitana (Girón, Piedecuesta y Floridablanca) que reportan al Ministerio de Protección Social, SISPRO, Supersalud.

\section{Materiales e instrumentos}

Se tomaron los resultados de 17 indicadores de IPS (Resolución 1446 de 2006), en los que se precisa el Sistema de Información para la Calidad y se acogen los indicadores de monitoreo y evaluación del Sistema 
Obligatorio de Garantía de Calidad de la Atención en Salud. Estos permiten medir los puntos críticos de la calidad en atención y servicios en salud del Ministerio de Salud de Colombia (MINSALUD, 2009, 2015).

\section{Tabla 1}

Indicadores de calidad desde la efectividad

\begin{tabular}{|c|c|c|}
\hline $\begin{array}{l}\text { Nombre del } \\
\text { indicador }\end{array}$ & Fórmula & $\begin{array}{l}\text { Unidad } \\
\text { de } \\
\text { medición }\end{array}$ \\
\hline $\begin{array}{l}\text { Tasa de reingreso } \\
\text { de pacientes } \\
\text { hospitalizados }\end{array}$ & $\begin{array}{l}\text { (Número total de pacientes que reingresan al servicio de } \\
\text { hospitalización, en la misma institución, antes de } 20 \text { días, } \\
\text { por la misma causa, en el periodo/número total de } \\
\text { egresos vivos en el período)* } 100\end{array}$ & $\begin{array}{l}\text { Relación } \\
\text { porcentual }\end{array}$ \\
\hline $\begin{array}{l}\text { Proporción de } \\
\text { pacientes con } \\
\text { hipertensión arterial } \\
\text { controlada (HTA) }\end{array}$ & $\begin{array}{l}\text { (Número total de pacientes que, seis meses después de } \\
\text { diagnosticada su hipertensión arterial, presentan niveles } \\
\text { de tensión arterial esperados, de acuerdo con las metas } \\
\text { recomendadas por la Guía de Práctica Clínica, basada en } \\
\text { evidencia/número total de pacientes hipertensos } \\
\text { diagnosticados)*100 }\end{array}$ & $\begin{array}{l}\text { Relación } \\
\text { porcentual }\end{array}$ \\
\hline $\begin{array}{l}\text { Tasa de mortalidad } \\
\text { intrahospitalaria } \\
\text { después de } 48 \\
\text { horas }\end{array}$ & $\begin{array}{l}\text { (Número total de pacientes que, seis meses después de } \\
\text { diagnosticada su hipertensión arterial, presentan niveles } \\
\text { de tensión arterial esperados, de acuerdo con las metas } \\
\text { recomendadas por la Guía de Práctica Clínica, basada en } \\
\text { evidencia/número total de pacientes hipertensos } \\
\text { diagnosticados) } 100\end{array}$ & $\begin{array}{l}\text { Relación } \\
\text { porcentual }\end{array}$ \\
\hline
\end{tabular}

Fuente: Autores.

Tabla 2

Indicadores de calidad desde la seguridad

\begin{tabular}{lll}
\hline Nombre del indicador & \multicolumn{1}{c}{ Fórmula } & \multicolumn{1}{c}{$\begin{array}{c}\text { Unidad de } \\
\text { medición }\end{array}$} \\
\hline $\begin{array}{l}\text { Tasa de infección } \\
\text { intrahospitalaria }\end{array}$ & $\begin{array}{l}\text { (Número de pacientes con infección nosocomial/número } \\
\text { total de pacientes hospitalizados) }\end{array}$ & $\begin{array}{l}\text { Relación } \\
\text { porcentual }\end{array}$ \\
$\begin{array}{l}\text { Proporción de vigilancia } \\
\text { de eventos adversos }\end{array}$ & $\begin{array}{l}\text { (Número total de eventos adversos detectados y } \\
\text { gestionados/número total de eventos adversos } \\
\text { detectados) } 100\end{array}$ & $\begin{array}{l}\text { Relación } \\
\text { porcentual }\end{array}$ \\
\hline
\end{tabular}

Fuente: Autores, a partir de la Resolución N. ${ }^{\circ} 1446$ de 8 mayo de 2006.

\section{Procesamiento y análisis de datos}

Los datos de indicadores de calidad fueron analizados mediante el software SPSS, herramienta de preferencia para analizar datos destinados a publicaciones como esta. Se utiliza la estadística descriptiva para cada uno de los indicadores de calidad.

\section{Resultados}

Los resultados de los indicadores de calidad en la atención de la salud en Bucaramanga y su área metropolitana desde la perspectiva de efectividad se presentan en la Tabla 4. Estos están directamente relacionados con la capacidad para resolver adecuadamente los problemas de salud en las entidades con servicios de hospitalización. 


\section{Tabla 3}

Indicadores de calidad desde la experiencia en la atención

\begin{tabular}{|c|c|c|}
\hline Nombre del indicador & Fórmula & $\begin{array}{l}\text { Unidad de } \\
\text { medición }\end{array}$ \\
\hline $\begin{array}{l}\text { Oportunidad de la } \\
\text { asignación de cita en la } \\
\text { consulta médica general (IPS) }\end{array}$ & $\begin{array}{l}\text { (Sumatoria total de los días calendario transcurridos entre la } \\
\text { fecha en la cual el paciente solicita cita para ser atendido en } \\
\text { la consulta médica general y la fecha para la cual es asignada } \\
\text { la cita/número total de consultas médicas generales } \\
\text { asignadas en la institución)^100 }\end{array}$ & Días \\
\hline $\begin{array}{l}\text { Oportunidad de la } \\
\text { asignación de cita en la } \\
\text { consulta médica } \\
\text { especializada (medicina } \\
\text { interna, ginecoobstetricia, } \\
\text { pediatría y cirugía general) }\end{array}$ & $\begin{array}{l}\text { (Sumatoria total de los días calendario transcurridos entre } \\
\text { la fecha en la cual el paciente solicita cita para ser atendido } \\
\text { en la consulta médica especializada y la fecha para la cual } \\
\text { es asignada la cita/número total de consultas médicas } \\
\text { especializadas asignadas en la institución) }\end{array}$ & Días \\
\hline $\begin{array}{l}\text { Oportunidad en la } \\
\text { realización de cirugía } \\
\text { programada }\end{array}$ & $\begin{array}{l}\text { (Sumatoria total de los días calendario transcurridos entre } \\
\text { la fecha de solicitud de la cirugía programada y el } \\
\text { momento en el cual es realizada la cirugía/número de } \\
\text { cirugías programadas realizadas en el período) }\end{array}$ & Días \\
\hline $\begin{array}{l}\text { Oportunidad en la atención } \\
\text { en consulta de odontología } \\
\text { general }\end{array}$ & $\begin{array}{l}\text { (Sumatoria total de los días calendario transcurridos entre } \\
\text { la fecha en la cual el paciente solicita cita para ser atendido } \\
\text { en la consulta de odontología general y la fecha para la } \\
\text { cual es asignada la cita/número total de consultas } \\
\text { odontológicas generales asignadas en el periodo en la } \\
\text { institución) }\end{array}$ & Días \\
\hline $\begin{array}{l}\text { Oportunidad en la atención } \\
\text { en servicios de imagenología }\end{array}$ & $\begin{array}{l}\text { (Sumatoria del número de días transcurridos entre la } \\
\text { solicitud del servicio de imagenología y el momento en el } \\
\text { cual es prestado el servicio/ total de atenciones en servicios } \\
\text { de imagenología) }\end{array}$ & Días \\
\hline $\begin{array}{l}\text { Proporción de cancelación } \\
\text { de cirugía programada }\end{array}$ & $\begin{array}{l}\text { (Número total de cirugías canceladas en el periodo/número } \\
\text { de cirugías programadas en el período) } 100\end{array}$ & $\begin{array}{l}\text { Relación } \\
\text { porcentual }\end{array}$ \\
\hline $\begin{array}{l}\text { Oportunidad en la atención } \\
\text { en consulta de urgencias }\end{array}$ & $\begin{array}{l}\text { (Sumatoria del número de minutos transcurridos entre la } \\
\text { solicitud de atención en la consulta de urgencias y el } \\
\text { momento en el cual es atendido el paciente en consulta por } \\
\text { parte del médico/total de usuarios atendidos en consulta } \\
\text { de urgencias) }\end{array}$ & Minutos \\
\hline Tasa de satisfacción global & $\begin{array}{l}\text { (Número total de pacientes que se consideran satisfechos } \\
\text { con los servicios recibidos por la IPS/número total de } \\
\text { pacientes encuestados por la IPS) }{ }^{*} 100\end{array}$ & $\begin{array}{l}\text { Relación } \\
\text { Porcentual }\end{array}$ \\
\hline
\end{tabular}

Fuente: Autores, a partir de la Resolución N. ${ }^{\circ} 1446$ de 8 mayo de 2006.

La tasa de reingreso de pacientes hospitalizados mide el ingreso en los 30 días siguientes al alta. La tasa media nacional de reingreso de pacientes fue del 1,45\% para el año 2015; a escala departamental, la tasa en los últimos cinco años se ha incrementado de $1,88 \%$, en 2009 , a $4,09 \%$, en 2015. De esta forma, Santander es uno de los departamentos con el indicador más alto, después de Caquetá, con $6,80 \%$, y Guaviare, con 4,86 \%. La tasa más alta se encuentra en Bucaramanga, que para el 2015 registró el 8,48 \%; seguida de Girón, con 1,47 \%; Floridablanca, con 1,15\%; y Piedecuesta, con 0,69 \%. Es importante que las instituciones hospitalarias planteen acciones efectivas para detectar las personas con riesgo de reingreso, mediante el desarrollo de intervenciones más efectivas y perdurables en el tiempo. 
La proporción de pacientes con hipertensión arterial controlada (HTA) permite identificar el cumplimiento de los objetivos conexos con la HTA, los tratamientos adecuados para reducir las complicaciones, las secuelas y la mortalidad. Los informes de pacientes hipertensos con presión arterial (PA) controlada han sido fluctuantes. A escala regional, la media de PA controlados pasó de $61,84 \%$ en 2011 a $51,61 \%$ en 2015 . Bucaramanga y su área metropolitana para el 2015 alcanzaron mejores resultados de PA controlados que la media nacional, que obtuvo un 63,24\%. El bajo porcentaje de este indicador se asocia con el restringido acceso a las instituciones de salud, el incumplimiento del tratamiento y una desmedida incidencia de hipertensión entre minorías étnicas y raciales.

La disminución de las muertes intrahospitalarias es un objetivo de las clínicas, los administradores y los pacientes. En Santander se observa una tasa global de mortalidad intrahospitalaria posterior a 48 horas, con un valor que fluctúa entre un rango mínimo de 1,7\%, en 2012, hasta de 2,5\%, en 2014. Para el 2015, se alcanzó una tasa de $2,0 \%$, superior a la media nacional, que fue de 1,3\%. Floridablanca y Bucaramanga, por término medio, registraron las tasas más altas durante el periodo evaluado. Esto se debe a que en estas regiones se encuentra ubicado el mayor número de clínicas y centros hospitalarios.

Tabla 4

Indicadores de calidad en la atención de la salud en Bucaramanga y su área metropolitana desde la perspectiva de efectividad

\begin{tabular}{lccccc}
\hline \multicolumn{5}{c}{ Tasa de reingreso de pacientes hospitalizados } \\
\hline Ciudad/región & $\mathbf{2 0 1 1}$ & $\mathbf{2 0 1 2}$ & $\mathbf{2 0 1 3}$ & $\mathbf{2 0 1 4}$ & $\mathbf{2 0 1 5}$ \\
Bucaramanga & 2,0 & 1,6 & 3,2 & 4,5 & 8,5 \\
Floridablanca & 2,0 & 1,2 & 1,5 & 1,2 & 1,2 \\
Girón & 0,7 & 2,3 & 0,6 & 0,6 & 1,5 \\
Piedecuesta & 0,5 & 0,0 & 0,1 & 0,7 & 0,7 \\
Santander & 1,9 & 2,0 & 2,7 & 4,3 & 4,1 \\
Proporción de pacientes con hipertensión arterial controlada \\
Bucaramanga & 71,8 & 85,3 & 69,4 & 77,6 & 68,5 \\
Floridablanca & 69,7 & 69,2 & 64,7 & 67,0 & 80,5 \\
Girón & 81,2 & \multicolumn{5}{c}{8,0} & 89,7 & 91,9 \\
Piedecuesta & 34,1 & 88,0 & 36,8 & 83,8 & 73,4 \\
Santander & 61,8 & 56,5 & 49,5 & 78,8 & 51,6 \\
\multicolumn{1}{r}{ Tasa de mortalidad intrahospitalaria después de $\mathbf{4 8}$ horas } \\
Bucaramanga & 2,7 & 2,9 & 1,5 & 1,6 & 2,4 \\
Floridablanca & 3,2 & 3,6 & 2,9 & 2,3 & 2,6 \\
Girón & 0,2 & 0,0 & 0,1 & 0,1 & 0,4 \\
Piedecuesta & 0,3 & 0,6 & 0,1 & 0,1 & 0,4 \\
Santander & 2,6 & 1,7 & 1,9 & 2,5 & 2,0 \\
\hline
\end{tabular}

Fuente: Autores.

\section{Indicadores de calidad desde la perspectiva de seguridad}

La seguridad en la atención en Colombia se ha desarrollado en los últimos años debido a la incorporación de políticas y destinación de recursos. La efectividad de los servicios de salud carece de sentido si no son seguros; o si se alcanzan sin analizar el riesgo o los resultados en la salud de las personas. Esto puede generar nuevos daños, discapacidades o lesiones.

En Santander la tasa de infección intrahospitalaria ha tenido una tendencia fluctuante y decreciente en el periodo 2011-2015, con 1,9\%, en 2015, superior a la media nacional, que fue de 0,99\%. El indicador más alto lo registraron Floridablanca y Bucaramanga, con tasas de $2,7 \%$ y $2,4 \%$, respectivamente. Otro indicador es la tasa de proporción de vigilancia de efectos adversos, que se asocia con la eficiencia, efectividad y calidad de la clínica. Santander presentó una tendencia decreciente en el periodo evaluado, pasando de 94,3\%, en 2011, a 19,6 \% en 2015 (ver Tabla 5).

\section{Tabla 5}

Indicadores de calidad desde la perspectiva de seguridad

\begin{tabular}{lccccc}
\hline \multicolumn{7}{c}{ Tasa de infección intrahospitalaria } \\
\hline Ciudad/región & $\mathbf{2 0 1 1}$ & $\mathbf{2 0 1 2}$ & $\mathbf{2 0 1 3}$ & $\mathbf{2 0 1 4}$ & $\mathbf{2 0 1 5}$ \\
Bucaramanga & 2,2 & 1,8 & 1,5 & 1,5 & 2,4 \\
Floridablanca & 3,0 & 4,2 & 2,0 & 2,2 & 2,7 \\
Girón & 0,0 & 0,0 & 0,0 & 0,1 & 0,2 \\
Piedecuesta & 0,0 & 0,0 & 0,0 & 0,2 & 0,1 \\
Santander & 1,9 & 3,3 & 1,6 & 2,1 & 1,9 \\
\multicolumn{5}{c}{ Proporción de vigilancia de eventos adversos } \\
Bucaramanga & 94,7 & 3,6 & 13,8 & 12,1 & 15,4 \\
Floridablanca & 92,7 & 95,5 & 25,7 & 88,7 & 83,7 \\
Girón & 93,4 & 96,0 & 96,0 & 75,4 & 73,2 \\
Piedecuesta & 98,3 & 50,0 & 100,0 & 97,6 & 95,2 \\
Santander & 94,3 & 8,3 & 16,9 & 17,3 & 19,6 \\
\hline
\end{tabular}

Fuente: Autores.

\section{Indicadores de calidad desde la perspectiva de experiencia en atención}

Los resultados del indicador de oportunidad de la asignación de citas en la consulta médica general (IPS) es importante, puesto que está relacionado con cuantiosos problemas de salud que pueden ser tratados sin trasladarse a los niveles superiores. Es necesario contar con tiempos de espera cortos en la asignación de citas, para resolver a tiempo las enfermedades, así como evitar el embotellamiento de los servicios de urgencias y consulta externa de los hospitales. Para Santander y los municipios de Bucaramanga y su área metropolitana 
se ha presentado un incremento en el tiempo de espera en días calendario para la asignación de cita. Para el año 2015, los municipios de Piedecuesta, con 3,2 días; Floridablanca, con 3,1 días; y Bucaramanga, con 2,9 días superaron la media nacional, que fue de 2,82 días. Es importante tener en cuenta que las falencias en este indicador de atención en salud están más relacionadas con factores de tipo institucional -como rigidez en los horarios, escaso personal, citas insuficientes, cuellos de botella de tipo administrativo- que con factores del usuario (ver Tabla 6).

Tabla 6

\section{Indicadores de calidad desde la perspectiva de experiencia en atención}

\begin{tabular}{|c|c|c|c|c|c|}
\hline \multicolumn{6}{|c|}{ Oportunidad de la asignación de citas en la consulta médica general } \\
\hline Ciudad/región & 2011 & 2012 & 2013 & 2014 & 2015 \\
\hline Bucaramanga & 1,4 & 1,3 & 2,2 & 2,5 & 2,9 \\
\hline Floridablanca & 2,5 & 3,6 & 3,5 & 3,1 & 3,1 \\
\hline Girón & 0,9 & 1,6 & 1,2 & 1,3 & 1,5 \\
\hline Piedecuesta & 2,7 & 4,0 & 2,5 & 2,0 & 3,2 \\
\hline Santander & 1,7 & 1,6 & 2,3 & 2,4 & 2,5 \\
\hline \multicolumn{6}{|c|}{ Oportunidad de la asignación de citas en la consulta de medicina interna } \\
\hline Bucaramanga & 12,3 & 14,7 & 16,1 & 14,2 & 14,3 \\
\hline Floridablanca & 13,9 & 9,1 & 12,7 & 14,9 & 13,9 \\
\hline Girón & & 9,7 & 3,5 & 3,1 & 3,6 \\
\hline Piedecuesta & 7,7 & 8,0 & 15,3 & 18,7 & 11,0 \\
\hline Santander & 11,0 & 11,1 & 13,6 & 13,2 & 13,3 \\
\hline \multicolumn{6}{|c|}{ Oportunidad de la asignación de citas en la consulta de ginecobstetricia } \\
\hline Bucaramanga & 3,9 & 2,9 & 10,3 & 10,5 & 8,4 \\
\hline Floridablanca & 13,4 & 9,1 & 10,1 & 10,4 & 13,1 \\
\hline Girón & 8,0 & 5,3 & 6,4 & 7,0 & 7,6 \\
\hline Piedecuesta & 3,3 & 10,0 & 10,1 & 6,9 & 7,9 \\
\hline Santander & 5,6 & 6,7 & 9,3 & 10,4 & 8,4 \\
\hline \multicolumn{6}{|c|}{ Oportunidad de la asignación de citas en la consulta de pediatría } \\
\hline Bucaramanga & 6,0 & 6,8 & 5,7 & 4,1 & 6,1 \\
\hline Floridablanca & 4,1 & 4,7 & 9,3 & 9,4 & 5,0 \\
\hline Girón & & 3,0 & 1,8 & 4,3 & 6,2 \\
\hline Piedecuesta & 3,4 & 4,0 & 5,0 & 4,0 & 4,2 \\
\hline Santander & 5,3 & 5,2 & 6,1 & 5,3 & 5,7 \\
\hline \multicolumn{6}{|c|}{ Oportunidad de la asignación de citas en la consulta de cirugía general } \\
\hline Bucaramanga & 7,4 & 8,8 & 14,9 & 11,9 & 13,3 \\
\hline Floridablanca & 10,1 & 5,1 & 6,6 & 5,4 & 7,8 \\
\hline Girón & & 11,5 & 4,0 & 4,7 & 12,3 \\
\hline Piedecuesta & 2,0 & 2,0 & 8,5 & 12,6 & 5,7 \\
\hline Santander & 6,9 & 7,2 & 12,5 & 11,0 & 11,9 \\
\hline \multicolumn{6}{|c|}{ Proporción de cancelación de cirugía programada } \\
\hline Bucaramanga & 6,1 & 2,8 & 9,2 & 11,4 & 7,8 \\
\hline Floridablanca & 5,1 & 5,7 & 4,3 & 5,2 & 5,4 \\
\hline Girón & & & & & \\
\hline Piedecuesta & 8,3 & 0,8 & 3,7 & 1,7 & 3,7 \\
\hline Santander & 5,7 & 6,6 & 7,5 & 10,0 & 7,3 \\
\hline \multicolumn{6}{|c|}{ Oportunidad de atención en consulta de urgencias } \\
\hline Bucaramanga & 46,6 & 26,9 & 42,2 & 40,1 & 52,6 \\
\hline Floridablanca & 43,0 & 40,3 & 36,8 & 33,9 & 51,3 \\
\hline Girón & 30,0 & 30,0 & 30,0 & 30,0 & 30,0 \\
\hline Piedecuesta & 28,3 & 14,2 & 28,5 & 28,5 & 30,9 \\
\hline Santander & 37,1 & 21,9 & 33,7 & 30,5 & 39,2 \\
\hline \multicolumn{6}{|c|}{ Oportunidad en la atención en servicios de imagenología } \\
\hline Bucaramanga & 2,1 & 2,6 & 3,7 & 9,5 & 5,8 \\
\hline Floridablanca & 3,2 & 5,8 & 4,6 & 22,9 & 11,6 \\
\hline Girón & 1,0 & 1,6 & 1,2 & 2,1 & 1,0 \\
\hline Piedecuesta & 1,7 & 1,2 & 2,0 & 3,0 & 1,6 \\
\hline Santander & 2,2 & 3,1 & 3,6 & 12,2 & 6,5 \\
\hline
\end{tabular}

\begin{tabular}{|c|c|c|c|c|c|}
\hline \multicolumn{6}{|c|}{ Oportunidad en la atención en consulta de odontología general } \\
\hline Bucaramanga & 1,8 & 1,2 & 2,2 & 3,1 & 3,5 \\
\hline Floridablanca & 2,9 & 2,6 & 2,8 & 2,9 & 2,9 \\
\hline Girón & 1,0 & 1,8 & 1,0 & 1,3 & 1,4 \\
\hline Piedecuesta & 5,1 & 2,0 & 3,2 & 2,9 & 3,6 \\
\hline Santander & 2,4 & 1,6 & 2,3 & 2,6 & 2,8 \\
\hline \multicolumn{6}{|c|}{ Oportunidad en la realización de cirugía programada } \\
\hline Bucaramanga & 13,1 & 15,8 & 22,7 & 21,5 & 21,3 \\
\hline Floridablanca & 9,4 & 4,5 & 10,7 & 12,1 & 5,4 \\
\hline \multicolumn{6}{|l|}{ Girón } \\
\hline Piedecuesta & 20,2 & & 16,6 & 7,2 & 11,2 \\
\hline Santander & 11,0 & 9,2 & 16,8 & 16,7 & 15,0 \\
\hline \multicolumn{6}{|c|}{ Tasa de satisfacción global } \\
\hline Bucaramanga & 95,2 & 95,4 & 92,5 & 95,2 & 93,2 \\
\hline Floridablanca & 82,0 & 96,1 & 75,2 & 92,6 & 90,4 \\
\hline Girón & 84,8 & 80,2 & 92,2 & 93,5 & 96,6 \\
\hline Piedecuesta & 95,7 & 95,1 & 94,3 & 71,0 & 72,0 \\
\hline Santander & 92,9 & 60,2 & 90,8 & 91,9 & 88,6 \\
\hline
\end{tabular}

Fuente: Autores.

El indicador oportunidad de atención en consulta de urgencias requiere una especial supervisión. Es el factor que más se vincula con la preservación de la vida frente a una amenaza cinco. Durante el período 2011-2015, se presentó una tendencia creciente, pasando a nivel de Santander, en 2011, de 37,1 min a 39,2 min, en 2015, mientras que la media nacional fue de $36,7 \mathrm{~min}$ para el mismo año. En 2015, Bucaramanga, con 52,6 min, y Floridablanca, con 51,3 min, fueron las regiones con mayor tiempo de espera. Estos tiempos podrían estar relacionados con factores como mayor demanda de la población a los servicios de urgencia por larga espera, consulta ambulatoria, insuficiencia de capacidad instalada, entre otros.

Finalmente, el Ministerio de la Protección Social propuso el indicador de tasa de satisfacción global, para medir la opinión de los pacientes sobre los servicios y trato recibido. Este indicador es una herramienta muy interesante que permite obtener información para optimizar los recursos disponibles y las necesidades de los usuarios. La satisfacción de los usuarios en todo el periodo fue variable. En Santander, para el año 2015, fue del 88,6 \%; en Girón, de 96,8 \%; en Bucaramanga, de 93,2 $\%$; y en Floridablanca, de $90,4 \%$, de modo que se superó la media nacional, que fue del $78,24 \%$.

\section{Comentarios}

Se encontraron problemáticas en la calidad del uso de los recursos destinados a la salud en Bucaramanga y su área metropolitana que están relacionados con la efectividad, la seguridad y experiencia de la atención.

Bucaramanga y Floridablanca, por término medio, son los municipios con mayores dificultades relacionadas con tasas de oportunidad en la asignación de citas médicas en las distintas especialidades, así como con las 
tasas de mortalidad de los indicadores evaluados. Esto se debe principalmente a las condiciones geográficas y la ubicación del mayor número de clínicas y centros hospitalarios.

Las falencias evidenciadas requieren acciones de mejora urgentes. Es necesario revisar los principales factores que ocasionaron los resultados, como factores de tipo institucional, rigidez en los horarios, escaso personal, citas insuficientes, cuellos de botella de tipo administrativo, falta de recursos, entre otros.

\section{Agradecimientos}

A la Universidad Autónoma de Bucaramanga, por la financiación del proyecto de investigación titulado Estimación de la eficiencia de la gestión pública en el sector salud, a partir de indicadores técnicos que evalúan la calidad de vida en Bucaramanga y su área metropolitana.

\section{Referencias}

Calderón, C. (2019). Atención primaria de salud: por qué, dónde y cómo. Hacia la Promoción de la Salud, 24(1), 9-10.

Cano, S. M., Pineda, Á. G., y Pulido, C. F. (2016). Concepto de calidad en salud: resultado de las experiencias de la atención, Medellín, Colombia. Facultad Nacional de Salud Pública: El escenario para la salud pública desde la ciencia, 34(1), 48-53.

CEPAL. (2018). Agenda 2030 y los Objetivos de Desarrollo Sostenible: una oportunidad para América Latina y el Caribe. Recuperado de https://repositorio.cepal. org/bitstream/handle/11362/40155/24/S1801141_ es.pdf

Charnes, A. (2013). Data envelopment analysis: theory, methodology, and application.

Chisholm, D., y Evans, D. B. (2010). Improving health system efficiency as a means of moving towards universal coverage. digicollection.orgWorld health report,. Recuperado de http://digicollection.org/ hss/documents/s18310en/s18310en.pdf

Cotlear, D., Gómez-Dantés, O., Knaul, F., Atun, R., Barreto, H. C., Ivana, C., y Guerrero, R. (2015). La lucha contra la segregación social en la atención de salud en América Latina. MEDICC Review, 17(S1), 40-52.

Diaz, M., Ospina, M., y Lopez, C. (2015). Cobertura relativa como falla esencial del Sistema de salud en Colombia. ISOCUANTA, 4(2). Recuperado de http:// revistas.usta.edu.co/index.php/isocuanta/article/ view/2420

Echeverri, O. (2008). Mercantilización de los servicios de salud para el desarrollo: el caso de Colombia.
Revista Panamericana de Salud Pública, 24, 210-216.

Hernández, J. M. R., Rubiano, D. P. R., y Barona, J. C. C. (2015). Barreras de acceso administrativo a los servicios de salud en población Colombiana. Ciência y Saúde Coletiva, 20, 1947-1958.

Kruk, M. E., Gage, A. D., Joseph, N. T., Danaei, G., GarcíaSaisó, S., y Salomon, J. A. (2018). Mortality due to low-quality health systems in the universal health coverage era: a systematic analysis of amenable deaths in 137 countries. The Lancet, In press, 392, 2203-2212.

MINSALUD. (2009). Informe Nacional de Calidad de la atención en Salud. Bogotá,. Recuperado de http:// calidadensalud.minsalud.gov.co/Paginas/Inicio. aspx

MINSALUD. (2015). Informe Nacional de Calidad de la atención en Salud. Bogotá.

Murray, C., y Frenk, A. (2000). Framework for assessing the performance of health systems. Bulletin of the world Health Organization, 78(6), 17-31.

Ramírez-Sánchez, y Nájera-Aguilar, D. T. (1998). Percepción de la calidad de la atención de los servicios de salud en México: perspectiva de los usuarios. Salud pública de México, 40, 03-12. Recuperado de https://www.scielosp.org/scielo.php?script=sci_ arttextypid=S0036-36341998000100002

Roldán, P., Vargas, C., y Giraldo, C. (2001). Evaluación de la calidad en la atención en salud. Un estudio de caso. Colombia Médica, 32(1). Recuperado de https://www. redalyc.org/html/283/28332104/

Ruiz, D., Duque, C. y Redondo, M. (2017). El TLC con la Unión Europea y sus implicaciones en el sector agrícola colombiano. (Spanish). Sinapsis: Revista de Investigaciones de la Escuela de Administración y Mercadotecnia del Quindío EAM, 9(1), 42. Recuperado de http://ezproxy.eafit.edu.co/login?url=http:// search.ebscohost.com/login.aspx?direct $=$ true $y d b=$ edby $A N=127554306$ lang $=$ esysite $=$ eds liveyscope $=$ site

UNESCO. (2005). Objetivos de desarrollo del milenio: Una mirada desde América Latina y el Caribe. (United Nations Publications. CEPAL, Ed.).

Zea, J. R., y Restrepo, V. S. (2002). El seguro obligatorio de salud en Colombia: evolución y determinantes de la cobertura. Recuperado de http://tesis.udea. edu.co/handle/10495/7341 